\title{
Clinical Outcomes in Carbapenem-Resistant Enterobacteriaceae Infections Treated With Ceftazidime-Avibactam: A Single-Center Observational Study
}

\author{
Balram Rathish ${ }^{1}$, Arun Wilson ${ }^{1}$, Anup Warrier ${ }^{1}$, Shilpa Prakash ${ }^{2}$, Rachana Babu ${ }^{3}$, Sonya Joy ${ }^{3}$ \\ 1. Infectious Diseases, Aster Medcity, Kochi, IND 2. Clinical Pharmacy, Aster Medcity, Kochi, IND 3. Clinical \\ Microbiology, Aster Medcity, Kochi, IND
}

Corresponding author: Arun Wilson, dr.arunwilson@gmail.com

\begin{abstract}
Introduction

Among the several newer beta lactam+beta lactase inhibitors (BL/BLI), ceftazidime-avibactam is the only drug showing activity against OXA-48-like producers. Hence, it is being increasingly used in India to treat infections caused by carbapenem-resistant Enterobacteriaceae (CRE), especially as a colistin-sparing agent. We have used ceftazidime-avibactam in patients suspected and confirmed to have CRE infections in our center, and present a retrospective analysis of our experience.
\end{abstract}

\section{Methods}

We conducted a single-center, retrospective study involving all patients who were treated with ceftazidimeavibactam for suspected and proven CRE infections during a one-year period at our 500-bedded hospital. Our primary objective for this study was taken as all-cause mortality. The secondary objectives were to determine the clinical cure, defined as the end of the treatment regimen with a resolution of primary infection and resistance to ceftazidime-avibactam in patients who underwent the Epsilometer test (E-test).

\section{Results}

A total of 103 patients who received ceftazidime-avibactam were identified. The all-cause mortality was $27 \%$ while a clinical cure was achieved in $73 \%$. Fifty-two patients received empirical therapy and 51 patients received ceftazidime-avibactam for confirmed CRE infection. Forty-eight patients had an E-test done, out of which $79 \%$ of patients had CREs sensitive to ceftazidime-avibactam, and $21 \%$ of patients had ceftazidimeavibactam resistant CREs. A higher Sequential Organ Failure Assessment (SOFA) score, Charlson comorbidity index (CCI) score, intensive care unit (ICU) admission, inotrope requirement, and lower days of therapy (DOT) with ceftazidime-avibactam were found to be associated with increased mortality.

Review began 01/21/2021 Review ended 01/24/2021 Published 02/02/2021

(c) Copyright 2021 Rathish et al. This is an open access article distributed under the terms of the Creative Commons Attribution License CC-BY 4.0., which permits unrestricted use, distribution, and reproduction in any medium, provided the original author and source are credited.

\section{Conclusion}

Colistin has been considered to be the last-line agent in CRE infections, but there are concerns about its adverse effects and the emergence of resistance. Given our relatively low mortality of $27 \%$ in CRE infections treated with ceftazidime-avibactam, coupled with the high susceptibility of the tested isolates, there may be a role for the empirical use of this drug in infections caused by CRE, especially in a setting where colistin may not be ideal.

Categories: Infectious Disease, Epidemiology/Public Health

Keywords: ceftazidime-avibactam, carbapenem resistant enterobacteriaceae, antimicrobial resistance, india

\section{Introduction}

Ceftazidime-avibactam is a relatively new, parenterally administered combination of third-generation cephalosporin ceftazidime and avibactam, a novel non- $\beta$-lactam $\beta$-lactamase inhibitor [1]. Combining ceftazidime with avibactam significantly (four to 1024 times minimum inhibitory concentration (MIC) reduction) improves its activity against most species of Enterobacteriaceae, as well as against Pseudomonas aeruginosa (approximately four times the MIC reduction) [2]. The combination of avibactam, however, does not increase the activity of ceftazidime against Acinetobacter species and most other anaerobic bacteria [2]. India reports high rates of multi-drug resistant bacteria with recent studies showing a high prevalence of carbapenem-resistant Enterobacteriaceae (CREs) with some studies reporting about three percent prevalence to other reports of up to 30\% prevalence in Klebsiella pneumoniae and Escherichia coli [3-4]. Molecular analysis of the mechanism of resistance reports suggested a uniform distribution of the NDM and OXA-48 genes, with co-expression of NDM + OXA-48 in a small fraction of patients but no KPC or VIM [4]. 
Ceftazidime-avibactam has a broad spectrum of activity against Ambler molecular class A narrow-spectrum beta-lactamases, class A extended-spectrum beta-lactamases (ESBLs), and class A serine carbapenemases including KPC [5]. It also has activity against some class C such as KPC and class D carbapenemases, including OXA-48-like enzymes that may be present in Enterobacteriaceae. However, it has no activity against Ambler class B encompassing the metallo-beta-lactamases [5]. Among the several newer beta lactam+ beta lactase inhibitors (BL/BLI), ceftazidime-avibactam is the only drug showing activity against OXA-48-like producers [6]. Hence, ceftazidime-avibactam is being increasingly used in India to treat infections caused by CREs, especially as a colistin-sparing agent [7]. We have used ceftazidime-avibactam as both empirical and targeted therapy in patients suspected and confirmed to have CRE infections in our center, and present a retrospective analysis of our experience.

\section{Materials And Methods}

We conducted a single-center, retrospective study of all patients who were treated with ceftazidimeavibactam for a CRE infection from any source in a one-year period between September 1, 2019, and August 31, 2020, at our 500-bedded tertiary care hospital. The inclusion criteria for this study included patients aged more than 18 years who had received at least two days of therapy (DOT) of ceftazidime-avibactam in patients with renal dysfunction where dose modification was required, or 48 hours in patients without renal dysfunction. Pregnant women, children, and patients who received the drug for less than two DOT were excluded from the study. The patients were identified through our antimicrobial stewardship database. Baseline and demographic characteristics of the patients were accessed through electronic medical records and assessed from the time of the index infection. A full review was exempted by the institutional review board, as it was a retrospective study.

Syndromes of infections were defined using the Centers for Disease Control and Prevention/National Healthcare Safety Network Surveillance Definitions for Specific Types of Infections [8]. Baseline comorbidities of the patients were captured using the Charlson comorbidity index (CCI) [9]. The severity of the present infection was assessed using the Sequential Organ Failure Assessment (SOFA) score [10]. Both empirical and targeted therapy with ceftazidime-avibactam was included. Empirical therapy was given for cases with a high risk of CRE infections. Targeted therapy was initiated for confirmed CRE infections. Ceftazidime-avibactam was a restricted-use antibiotic in our center and the prescription of the agent was only done by authorized members of the antimicrobial stewardship team. Dosing was determined by the manufacturer's recommendation and the standard regimen for a CRE infection was taken as 2.5 grams thrice a day for 10 to 14 days. Concomitant therapy with antimicrobial spectrum against CRE infections was also recorded. The concomitant agent choice and dosing were prescriber specific.

Carbapenem resistance was measured using VITEK ${ }^{\circledR} 2$ (BioMérieux, Marcy-l'Étoile, France) antimicrobial susceptibility (AST) cards for determining the MIC of the studied CREs against meropenem and ertapenem and confirmed with the disc diffusion method. Carbapenem resistance was defined as resistance to any carbapenem using current Clinical and Laboratory Standards Institute (CLSI) breakpoints [11]. Patients underwent AST of ceftazidime-avibactam, which was done using non-commercial Epsilometer tests (E-tests) based on availability at the time.

Our primary objective for this study was taken as all-cause mortality. The secondary objectives were to determine the clinical cure, defined as the end of the treatment regimen with a resolution of primary infection, and resistance to ceftazidime-avibactam in patients who underwent the E-test. Patient characteristics were described using descriptive statistics. Group comparisons were made using unpaired ttests, Pearson's test, or Fisher's exact test. A p-value of $<0.05$ was considered significant.

\section{Results}

During the study period, a total of 141 patients received ceftazidime-avibactam. Out of these, 103 patients fulfilled the pre-determined inclusion criteria. The all-cause mortality was $27 \%(n=28)$ while a clinical cure was achieved in $73 \%(n=75)$. The comparisons between outcomes are summarized in Table 1 . The age of the patients ranged between 19 to 90 years of age with the mean (M), standard deviation (SD), median, and mode as $53.2 \pm 17.3,52$, and 45 years, respectively. There was no significant association between ages in mortality groups $(M=54.4, S D=17.6)$ and clinical cure groups $(M=52.7, S D=17.2)(t=0.4368, p=0.331)$. There were $78.5 \%(n=81)$ male patients and $21.5 \%(n=22)$ female patients. There was no significant association between sex and mortality $(\mathrm{p}=0.596)$. 


\section{Cureus}

\begin{tabular}{|c|c|c|c|c|}
\hline Patient characteristics & Total $(n=103)$ & Mortality (n=28) & Clinical cure $(n=75)$ & p-value \\
\hline Male sex & 81 & 21 & 60 & 0.596 \\
\hline Age $^{1}$ & $53.2 \pm 17.3$ & $54.4 \pm 17.6$ & $52.7 \pm 17.2$ & 0.331 \\
\hline SOFA score & $4.3 \pm 3.2$ & $5.7 \pm 3.5$ & $3.8 \pm 2.9$ & 0.004 \\
\hline CCI Score ${ }^{1}$ & $4.1 \pm 2.3$ & $5 \pm 2.4$ & $3.8 \pm 2.2$ & 0.008 \\
\hline ICU admission & 50 & 19 & 31 & 0.025 \\
\hline Inotrope requirement & 30 & 15 & 15 & 0.001 \\
\hline Ventilatory requirement & 46 & 16 & 30 & 0.127 \\
\hline Targeted therapy & 51 & 10 & 41 & 0.120 \\
\hline Renal dose modification & 26 & 8 & 18 & 0.803 \\
\hline Ceftazidime-avibactam DOT & $8.1 \pm 4.3$ & $1 \pm 3.8$ & $8.0 \pm 4.4$ & 0.050 \\
\hline Monotherapy & 69 & 20 & 49 & 0.642 \\
\hline Culture growth & 63 & 19 & 44 & 0.497 \\
\hline E-test sensitive & 38 & 10 & 28 & 1 \\
\hline
\end{tabular}

\section{TABLE 1: Comparison of characteristics between the two outcome groups}

NA: Not Applicable, SOFA: Sequential Organ Failure Assessment, CCl: Charlson Comorbidity Index, DOT: Days of Therapy

\footnotetext{
${ }^{1}$ Expressed as mean \pm standard deviation
}

The SOFA scores ranged between 0 to 16 with a mean, standard deviation, median, and mode scores of $4.3 \pm 3.2,4$, and 2 respectively. The SOFA score in the mortality group $(\mathrm{M}=5.7, \mathrm{SD}=3.5)$ was significantly higher than that of the clinical cure group $(\mathrm{M}=3.8, \mathrm{SD}=2.9)(\mathrm{t}=2.69606, \mathrm{p}=0.004)$. The $\mathrm{CCI}$ scores ranged between 0 and 10 with a mean, standard deviation, median, and mode scores of $4.1 \pm 2.3,4$, and 3 , respectively. The $\mathrm{CCI}$ score in the mortality group $(\mathrm{M}=5, \mathrm{SD}=2.4)$ was significantly higher than in the clinical cure group $(\mathrm{M}=3.8, \mathrm{SD}=2.2)(\mathrm{t}=3.18597, \mathrm{p}=0.008)$. Among the studied patients, there was also a positive correlation between the SOFA and CCI scores $(\mathrm{r}=0.29, \mathrm{p}=0.002)$.

Forty-eight point five percent $(48.5 \%$; $\mathrm{n}=50$ ) patients required intensive care unit (ICU) admission. There was a significant positive association between ICU admission and mortality $(\mathrm{p}=0.025)$. Twenty-nine percent $(29 \%$; $n=30)$ of patients were in shock, requiring inotropic support. There was a significant positive association between inotrope requirement and mortality $(\mathrm{p}=0.001)$. Forty-four point five percent $(44.5 \%$; $\mathrm{n}=46$ ) patients required ventilatory support. There was no association identified between ventilatory requirement and mortality $(\mathrm{p}=0.127)$.

Fifty percent $(50 \%$; $\mathrm{n}=52)$ of patients received empirical ceftazidime-avibactam, that is, it was started before the isolation of an organism while $50 \%(n=51)$ patients had the drug started based on the microbiological culture identification of a CRE. There was no significant difference in mortality or clinical cure identified between empirical or targeted therapy ( $35 \%$ vs $20 \%)(p=0.120)$. Although 52 patients were empirically initiated on ceftazidime-avibactam, over the course of the study, 12 patients were subsequently proven to have a CRE infection with the isolation of the organism on culture. Thirty-eight percent (38\%; $n=40)$ of patients did not have a positive microbiological culture throughout the course of their illness.

Out of the $62 \%(n=63)$ patients with a positive microbiological culture, $48 \%(n=49)$ had an infection with K.pneumoniae, $6 \%(n=6)$ had E.coli, $4 \%(n=4)$ patients had P.aeruoginosa while $4 \%(n=4)$ had mixed bacterial growth. Clinically, $48 \%(n=50)$ had bacteremia, $16 \%(n=16)$ had pneumonia, $10 \%(n=11)$ had an intraabdominal infection, $10 \%(\mathrm{n}=11)$ had a central nervous system (CNS) infection, $9 \%(\mathrm{n}=8)$ had a urinary tract infection (UTI), and 7\% (n=7) had a skin and soft tissue infection (SSTI). There was no significant mortality difference identified between culture positive patients and culture negative patients (30\% vs $22.5 \%$ ) $(\mathrm{p}=0.497)$.

Twenty-five percent $(25 \%$; $=26$ ) of patients had deranged renal functions and required renal dose modification of ceftazidime-avibactam. There was no association identified between renal dose modification 
or mortality ( $\mathrm{p}=0.803$ ). The DOT of ceftazidime-avibactam ranged between two to 22 days, with a mean, standard deviation, median, and mode of $8.1 \pm 4.3$, 7 , and 7 days, respectively. There was no significant association between the day of initiation of ceftazidime-avibactam and mortality. Sixty-seven percent (67\%; $\mathrm{n}=69)$ patients had monotherapy with ceftazidime-avibactam while $33 \%(n=34)$ had combination therapy with concomitant antibiotics with gram-negative coverage, including tigecycline, minocycline, polymixin, meropenem, levofloxacin, and aztreonam. There was no association identified between monotherapy or combination therapy with mortality ( $29 \%$ vs $23.5 \%)(\mathrm{p}=0.642)$.

Forty-six percent $(46 \% ; n=48)$ patients had an E-test done, out of which $79 \%(n=38)$ patients had CREs sensitive to ceftazidime-avibactam and $21 \%(n=10)$ patients had ceftazidime-avibactam-resistant CREs. There was no association identified between E-test sensitivity and mortality $(\mathrm{p}=1)$.

\section{Discussion}

South-Asia has become the hotbed of antimicrobial resistance, with some studies from India reporting a prevalence ranging from a modest $3 \%$ to an extremely high rate of around $65 \%$ CREs in clinical isolates [34,12-13]. Tigecycline and colistin are used as agents of choice for the treatment of CRE infections but treatment is complicated by uncertainties regarding the efficacy and adverse effects [14]. Newer agents like ceftazidime-avibactam have been increasingly used, as availability becomes wider with good clinical outcomes, however, data on clinical outcomes of ceftazidime-avibactam use in CREs is scarce from the Indian sub-continent. The mortality associated with CRE infections is higher than non-CRE infections but varies based on numerous factors [15-16]. Our study demonstrated overall mortality of $27 \%$, which is similar to the $32 \%$ mortality reported in another similar multi-centric study from the USA on the use of ceftazidimeavibactam in CREs by King et al. [17]. A study by Shields et al. found 30-day mortality of $24 \%$ in CREs treated with ceftazidime-avibactam [18]. However, Alraddadi et al. found a 50\% mortality in patients with CRE infections who received ceftazidime-avibactam from Saudi Arabia, highlighting the non-uniformity of mortality across geographical regions and patient characteristics [19].

A study on the clinical outcomes in patients receiving colistin by Prasannan et al., from our own center, found a mortality rate of around $10 \%$ in a sample of 150 patients who had received the drug [20]. However, they also found that almost $40 \%(n=59)$ of patients developed an acute kidney injury at some point during the colistin course. In our study, no patients were identified to have developed an AKI secondary to ceftazidimeavibactam. However, the other adverse drug events, including gastrointestinal manifestations, which are most commonly associated with ceftazidime-avibactam, were not available due to the retrospective nature of the study. Prasannan et al. only enrolled patients who had isolates susceptible to colistin and, hence, the resistance rates were not reported in the study, and the clinical details of patients who had colistin-resistant isolates were also not studied.

We also found significant positive associations between the initial SOFA scores and CCI scores of the patients and mortality. This is in keeping with previously published literature of worse outcomes in higher SOFA and higher CCI scores [9-10]. Mortality was significantly higher in patients who required ICU admissions and those who required inotropic support, which was in keeping with the high SOFA scores. However, we did not find an association between the requirement of ventilatory support and all-cause mortality. This could have been the result of unknown confounders or a result of an inadequate sample size.

In patients who underwent an E-test, almost $80 \%$ of CREs were sensitive to ceftazidime-avibactam. This was similar to the $73 \%$ reported in a large study by Spiliopoulou et al., but studies on resistance to ceftazidimeavibactam from clinical isolates were found to be few [21]. However, we failed to demonstrate a significantly different outcome in terms of mortality in patients with E-strip reported as sensitive, but this could have been as a result of the low number of patients who underwent the E-test. It could also have been due to a degree of sampling bias, where due to the limited availability of the E-test kits, only the most serious cases likely underwent the E-test, thereby further confounding the results.

Our study did have its limitations. We did not study the AST pattern of the isolates to colistin and other agents used for the treatment of CRE infections. Since this study was conducted retrospectively, parameters such as the SOFA score at the time of discharge or the adverse-effect profile of the drug could not be studied. Not all isolates underwent ceftazidime-avibactam AST due to the lack of a commercially available test. We were also not able to study the mechanism of resistance to ceftazidime-avibactam due to the widespread unavailability of molecular testing. The primary outcome chosen was all-cause mortality, and infection specific mortality could not be studied. There could also have been other confounders that were not accounted for.

\section{Conclusions}

Colistin has been considered to be the last-line agent in CRE infections, but there are increasing concerns about its adverse effects, and the emergence of colistin resistance. Given our relatively low mortality of $27 \%$ in CRE infections treated with ceftazidime-avibactam, coupled with the relatively high susceptibility of the tested isolates to it, there may be a role for the empirical use of this drug in infections caused by CRE, especially in a setting of existing renal disease where colistin may not be ideal. We used ceftazidime- 
avibactam as a potential colistin-sparing agent in view of its relatively safer adverse-effect profile. The better adverse effect profile and similar outcomes associated with the use of ceftazidime-avibactam can support its use as the first-line therapy in CREs and accentuate its role as a colistin-sparing agent.

\section{Additional Information \\ Disclosures}

Human subjects: Consent was obtained or waived by all participants in this study. Animal subjects: All authors have confirmed that this study did not involve animal subjects or tissue. Conflicts of interest: In compliance with the ICMJE uniform disclosure form, all authors declare the following: Payment/services info: All authors have declared that no financial support was received from any organization for the submitted work. Financial relationships: All authors have declared that they have no financial relationships at present or within the previous three years with any organizations that might have an interest in the submitted work. Other relationships: All authors have declared that there are no other relationships or activities that could appear to have influenced the submitted work.

\section{References}

1. Shirley M: Ceftazidime-avibactam: a review in the treatment of serious gram-negative bacterial . Drugs. 2018, 78:675-692. 10.1007/s40265-018-0902-x

2. Zhanel GG, Lawson CD, Adam H, et al.: Ceftazidime-avibactam: a novel cephalosporin/ $\beta$-lactamase inhibitor combination. Drugs. 2013, 73:159-177. 10.1007/s40265-013-0013-7

3. Sekar R, Srivani S, Amudhan M, Mythreyee M: Carbapenem resistance in a rural part of southern India: Escherichia coli versus Klebsiella spp. Indian J Med Res. 2016, 144:781-783. 10.4103/ijmr.IJMR 103515

4. Jaggi N, Chatterjee N, Singh V, Dwivedi P, Panwar R, Sharma AP: Carbapenem resistance in Escherichia coli and Klebsiella pneumoniae among Indian and international patients in North India. Acta Microbiol Immunol Hung. 2019, 66:367-376. 10.1556/030.66.2019.020

5. Toussaint KA, Gallagher JC: $\beta$-Lactam/ $\beta$-lactamase inhibitor combinations: from then to now . Ann Pharmacother. 2015, 49:86-98. 10.1177/1060028014556652

6. Pragasam AK, Veeraraghavan B, Shankar BA, et al.: Will ceftazidime/avibactam plus aztreonam be effective for NDM and OXA-48-Like producing organisms: lessons learnt from in vitro study. Indian J Med Microbiol. 2019, 37:34. 10.4103/ijmm.IJMM_19_189

7. Veeraraghavan B, Pragasam AK, Bakthavatchalam YD, Anandan S, Swaminathan S, Sundaram B: Colistinsparing approaches with newer antimicrobials to treat carbapenem-resistant organisms: current evidence and future prospects. Indian J Med Microbiol. 2019, 37:72. 10.4103/ijmm.IJMM 19215

8. CDC/NHSN surveillance definitions for specific types of infections . (2017). Accessed: December 15, 2020: https://www.cdc.gov/nhsn/pdfs/pscmanual/17pscnosinfdef_current.pdf.

9. Charlson ME, Pompei P, Ales KL, MacKenzie CR: A new method of classifying prognostic comorbidity in longitudinal studies: development and validation. J Chronic Dis. 1987, 40:373-383. 10.1016/00219681(87)90171-8

10. Jones AE, Trzeciak S, Kline JA: The Sequential Organ Failure Assessment score for predicting outcome in patients with severe sepsis and evidence of hypoperfusion at the time of emergency department presentation. Crit Care Med. 2009, 37:1649-1654. 10.1097/CCM.0b013e31819def97

11. Clinical and Laboratory Standards Institute (CLSI). Performance standards for antimicrobial susceptibility testing M100. (2018). Accessed: December 15, 2020: https://clsi.org/media/1930/m100ed28_sample.pdf.

12. Singh PK: One health approach to tackle antimicrobial resistance in South East Asia . BMJ. 2017, $358: j 3625$. 10.1136/bmj.j3625

13. Govindaswamy A, Bajpai V, Khurana S, Aravinda A, Batra P, Malhotra R, Mathur P: Prevalence and characterization of beta-lactamase-producing Escherichia coli isolates from a tertiary care hospital in India. J Lab Physicians. 2019, 11:123-127. 10.4103/JLP.JLP 12218

14. Doi Y: Treatment options for carbapenem-resistant gram-negative bacterial infections . Clin Infect Dis. 2019, 69:565-575. 10.1093/cid/ciz830

15. Balkhair A, Al-Muharrmi Z, Al'Adawi B, et al.: Prevalence and 30-day all-cause mortality of carbapenem-and colistin-resistant bacteraemia caused by Acinetobacter baumannii, Pseudomonas aeruginosa, and Klebsiella pneumoniae: description of a decade-long trend. Int J Infect Dis. 2019, 85:10-15. 10.1016/j.ijid.2019.05.004

16. Borer A, Saidel-Odes L, Riesenberg K, et al.: Attributable mortality rate for carbapenem-resistant Klebsiella pneumoniae bacteremia. Infect Control Hosp Epidemiol. 2009, 30:972-976. 10.1086/605922

17. King M, Heil E, Kuriakose S, et al.: Multicenter study of outcomes with ceftazidime/avibactam in patients with carbapenem-resistant Enterobacteriaceae (CRE) infections: a multi-center study. Antimicrob Agents Chemother. 2016, 61:e00449. 10.1128/AAC.00449-17

18. Shields RK, Potoski BA, Haidar G, et al.: Clinical outcomes, drug toxicity, and emergence of ceftazidimeavibactam resistance among patients treated for carbapenem-resistant Enterobacteriaceae infections. Clin Infect Dis. 2016, 63:1615-1618. 10.1093/cid/ciw636

19. Alraddadi BM, Saeedi M, Qutub M, Alshukairi A, Hassanien A, Wali G: Efficacy of ceftazidime-avibactam in the treatment of infections due to carbapenem-resistant Enterobacteriaceae. BMC Infect Dis. 2019, 19:772. 10.1186/s12879-019-4409-1

20. Prasannan B, Mukthar F, Unni V, et al.: Colistin nephrotoxicity - age and baseline kidney function hold the key [IN PRESS]. Indian J Nephrol. 2020,

21. Spiliopoulou I, Kazmierczak K, Stone GG: In vitro activity of ceftazidime/avibactam against isolates of carbapenem-non-susceptible Enterobacteriaceae collected during the INFORM global surveillance programme (2015-17). J Antimicrob Chemother. 2020, 75:384-391. 10.1093/jac/dkz456 